\title{
Fairnessnormen, Sanktionsmacht und soziale Kontrolle bei Bonuszahlungen in Unternehmen
}

\author{
Ein Multi-Level Factorial Survey Experiment
}

\section{Von Martin Wienhold und Knut Petzold*}

Zusammenfassung: Experimentelle Ergebnisse zu Verteilungssituationen zeigen, dass die Bereitschaft, einem anderen etwas zu geben, von einer Reihe soziologischer Faktoren abhängt und sowohl von eigeninteressierten als auch sozialen Motiven bestimmt wird. In dieser Arbeit wird in einem faktoriellen Survey-Design untersucht, wie Arbeitgeber kleiner und mittlerer Unternehmen in Deutschland 10.000 Euro zwischen sich und fiktiven Arbeitnehmern, welche bezüglich verschiedener Merkmale variieren, aufteilen würden. Im Fokus stehen die relative Stärke von Fairnessnormen und der Einfluss des Sanktionspotentials der Arbeitnehmer auf die Bonuszahlung sowie deren Beeinflussung durch unterschiedliche Informationsbedingungen. Dabei werden die Probanden zufällig einer von drei Entscheidungsumwelten zugeteilt, die sich im Grad der Transparenz der Verteilungssituation unterscheiden. Das Wissen der Arbeitnehmer darüber, dass es Anlass für einen Bonus gibt und das Wissen, wie viel einzelne Arbeitnehmer erhalten, aktiviert weitere Motive für eine Bonuszahlung hinzu und schwächt/stärkt Sanktionsmotive und Fairnessnormen verschieden. Die Fairnessnorm einer proportionalen Zahlung dominiert in allen Situationen. Doch deren Grad variiert stark zwischen Intransparenz und Transparenz der Verteilungssituation, was die Divergenz zwischen sozial erwünschten Erwartungen und subjektiven Fairnessnormen deutlich macht. Der Einfluss von Sanktionspotential der Arbeitnehmer verschwindet unter kompletter Transparenz. Insgesamt zeigt sich, dass das Vorhandensein sozialer Kontrolle nicht allein die Höhe des Bonus substantiell bestimmt, sondern auch die Kriterien, nach denen dieser verteilt wird.

\section{Einleitung}

Die Analyse von Verteilungssituationen kann interessante Einsichten in die Komplexität menschlichen Sozialverhaltens bieten. Bei der Frage danach, wie eine Ressource auf einzelne Individuen verteilt werden soll, kommen soziologische Kategorien wie Gerechtigkeit, Besitzanspruch, Legitimität, Devianz, Gruppenzugehörigkeit etc. zur Geltung. Sowohl die Frage nach Gerechtigkeitsprinzipien (z.B. Mill 1863; Rawls 1957) als auch die Frage danach, was Menschen überhaupt dazu bewegt, sich zum Vor- oder Nachteil der Mitmenschen zu verhalten (Hobbes 1651; Hume 1739; Smith 1759), sind grundlegende Fragen der Sozialtheorie und demnach auch für die aktuelle Sozialwissenschaft von hohem Interesse.

Dabei bietet besonders der verhaltensökonomische Ansatz die Möglichkeit, mit präzisen Theoriemodellen und der rigorosen Überprüfung mittels Experimenten, kumulativ wachsende Einsichten in menschliche und gesellschaftliche Fragestellungen zu liefern (Camerer / Fehr 2009; Diekmann 2008; Falk / Heckman 2009; Fehr / Gintis 2007). In zahlreichen Experimenten zu Verteilungssituationen werden immer wieder freiwillige Abgaben oder Hilfeleistungen beobachtet. Dabei zeigt sich, dass dieses Verhalten u.a. von soziologischen Faktoren wie von sozialem Status (Liebe / Tutic 2010), Gruppenzugehörigkeiten (Charness et al. 2007; Yamagishi / Mifune 2008) und Besitzansprüchen (Hoffman / Spitzer 1985; Oxoby / Spraggon 2006) abhängig ist. Typischerweise wird dieses Verhalten mit Fairnessmotiven

* Wir bedanken uns sehr für die konstruktiven und hilfreichen Kommentare der anonymen Gutachter und der Herausgeber dieser Zeitschrift zu einer früheren Version des Manuskripts.. 
(Cook / Hegtvedt 1983) oder auch Ungleichheitsaversion ${ }^{1}$ (Fehr / Schmidt 1999) begründet. Die Fairnessmotive, die in einer Verteilungssituation zum Tragen kommen, können dabei sehr unterschiedlich sein. In den Sozialtheorien zu Verteilungsgerechtigkeit wird neben dem Egalitarismus (equality) und der Leistungsgerechtigkeit (equity) noch zwischen den Prinzipien der Bedarfsgerechtigkeit, der kollektiven Wohlfahrt bzw. dem Utilitarismus und der Verantwortlichkeit bzw. Zurechenbarkeit von Leistungen unterschieden (z.B. Hegtvedt 1992; Konow 2003; Pointner 2012). Häufig angewandte ökonomische Modellierungen von Nutzenfunktionen, die einen variablen Fairnessterm enthalten, gehen dabei stets von einer Referenzverteilung aus, die sie für alle Akteure als die faire Verteilung unterstellen. So ist es in dem Modell der Ungleichheitsaversion nach Fehr und Schmidt (1999) die Gleichverteilung und in einer Erweiterung dieses Modells nach Tutic und Liebe (2009) die proportionale Verteilung, definiert über den relativen Status der Akteure.

Aber es ist zunächst eine empirische Frage, welches Fairnessprinzip tatsächlich zur Geltung kommt. Cappelen et al. (2007) untersuchen in einem Verteilungsspiel, in welchem die Probanden vorher in einer Produktionsphase einen bestimmten Betrag verdienen konnten, den sie dann gemeinsam mit dem Verdienst des anderen zwischen sich aufteilen, welche Fairnessprinzipien dominieren. Der Erfolg der Produktionsphase hängt dabei zum einen von nicht kontrollierbaren Bedingungen und zum anderen von der individuellen Anstrengung ab. Es zeigt sich eine gewisse Heterogenität von Gerechtigkeitsprinzipien, in welcher weder die reine Leistungsnorm noch die egalitäre Fairnessnorm dominiert. Stattdessen ist es eine differenziertere Fairnessnorm, in welchen die Probanden nur insofern für ihren Produktionserfolg verantwortlich gemacht werden, als dieser mit individueller Anstrengung zu beeinflussen ist. Fischbacher et al. (2009) berichten vergleichbare Ergebnisse aus einem ähnlichen Verteilungsspiel. Die Erklärung von Verteilungen mit Fairnessnormen, welche die Probanden in einer gegebenen Situation selber für angemessen halten, reicht dabei aber nicht aus. Es sind auch soziale Normen, durch die sich Leute zu sozialem Verhalten gezwungen fühlen, um in den Augen der anderen z.B. nicht als Egoist dazustehen. Dass soziale Kontrolle dabei einen Einfluss auf die Höhe der Abgaben hat, ist nachgewiesen (z.B. Cherry et al. 2002; Franzen / Pointner 2012; Hoffman et al. 1996). Der Druck normativen Erwartungen gerecht zu werden beschränkt sich allerdings nicht allein auf die Höhe der Abgabe, sondern kann genauso das Verteilungskriterium beeinflussen. Dies bedeutet, dass unter sozialer Kontrolle mit der Allokation einer Ressource eine Fairnessnorm signalisiert werden kann und diese nicht unbedingt den subjektiven Gerechtigkeitsvorstellungen entsprechen muss.

Genau diese mögliche „Verzerrung“ einer beobachtbaren Fairnessnorm durch variierende soziale Kontrolle untersuchen wir, indem wir mittels eines faktoriellen Surveys (Jasso 2006; Rossi / Anderson 1982; Rossi 1979) Geschäftsführer kleiner und mittlerer Unternehmen in Deutschland in unterschiedlich transparente Entscheidungssituationen versetzen, in welcher sie einen fiktiven Bonusbetrag von 10.000 Euro zwischen sich und einem Arbeitnehmer aufzuteilen haben. Wir argumentieren, dass eine zunehmende Transparenz die Allokation dadurch beeinflusst, indem weitere Motive wie die Erfüllung sozial erwünschter Erwartungen „hinzugeschaltet“" werden, was sowohl die Höhe der Bonuszahlung als auch die Verteilungskriterien mitbestimmen kann. Die zu verteilende Ressource stammt dabei aus dem gemeinsam produzierten Unternehmensgewinn. Die Arbeitnehmer unterscheiden sich unter anderem darin, dass sie unterschiedlich lang an der Produktion teilnehmen, dass sie unterschiedlich effizient sind, oder dass sie verschiedene Funktionen in dem arbeitsteiligen Prozess einnehmen. Da in diesem Kontext einer Bonuszahlung prinzipiell auch strategische Motive als

1 In der englischsprachigen Literatur wird häufig die passendere Bezeichnung „other-regarding preferences" verwendet. 
Reaktion auf die Sanktionsmacht der Arbeitnehmer eine Rolle spielen können, prüfen wir zugleich, welchen relativen Effekt Fairnessmotive und Sanktionsmacht in den unterschiedlichen Informationsbedingungen haben.

\section{Theoretische Überlegungen und Hypothesen}

Die experimentelle Untersuchung von Verteilungssituationen erfolgt typischerweise mit dem Diktator- und dem Ultimatum-Spiel (Camerer 2003; Güth et al. 1982; Kahneman et al. 1986), welche auch den heuristischen Rahmen unseres Designs bilden. Das Diktator-Spiel (DS) stellt dabei eine Verteilungssituation dar, in welcher allein ein Geber über die Aufteilung einer Ressource zwischen sich und mindestens einer weiteren Person, dem Empfänger, zu bestimmen hat. Im Gegensatz dazu besitzt im Ultimatum-Spiel (US) der Empfänger ein Vetorecht, welches dieser einsetzen kann, wenn ihm ein vom Geber vorgeschlagener Betrag zu niedrig erscheint. Im Falle eines Vetos erhalten dann beide nichts. Von besonderer Bedeutung in den vielfältigen Variationen dieser beiden Spiele ist die Herkunft der Ressource. Diese wird dabei entweder vom Experimentalleiter bereitgestellt (,windfall gain“) oder die Probanden müssen sich diese über eine Aufgabe, wie z.B. ein Quiz, verdienen (,earned wealth“). Das macht dabei einen großen Unterschied. So zeigen Ergebnisse aus DiktatorSpielen, dass Verteilende nichts abgeben, wenn die Ressource selbst verdient werden musste (z.B. Cherry et al. 2002 unter doppelblinden Bedingungen, also unter Anonymität zwischen den Probanden und zwischen Proband und Experimentalleiter). Zugleich steigen die Abgaben bis zu über 50\%, wenn der Empfänger die Ressource verdient hat (Oxoby / Spraggon 2008). Die Verteilungssituation wird also wesentlich dadurch bestimmt, wie die Eigentumsrechte bzw. die Besitzansprüche beschaffen sind (Feng et al. 2013; Hoffman / Spitzer 1985). In unserer Studie stammt die zu verteilende Ressource aus dem gemeinsam produzierten und obendrein unerwartet hohen Unternehmensgewinn. Allein dadurch ist nach dem Prinzip der Reziprozität (Gouldner 1960; Homas 1961) zu erwarten, dass der Arbeitgeber einen Bonus an seine Arbeitnehmer zahlt. Zusätzlich kann der Arbeitgeber einen Bonus auszahlen, um seine Angestellten zu motivieren. ${ }^{2}$ Diese prinzipielle Aktivierung der Reziprozitätsnorm schließt dabei die Untersuchung von Formen des Altruismus aus, wie z.B. den „warm-glow“ (Andreoni 1990) oder auch Empathie-Effekte. Stattdessen kann sowohl die Höhe als auch das Verteilungsmuster von sogenannten extrinsischen Motiven bzw. Mechanismen (Pointner / Franzen 2013; Pointner 2012) bestimmt werden. Damit werden Motive wie die Erfüllung sozial erwünschter Erwartungen, der Aufbau einer günstigen Reputation oder von Prestige bezeichnet (z.B. Willer 2009). Dies sind letztlich eigeninteressierte Motive, da sie einerseits dazu dienen, Sanktionen gegen einen selbst zu vermeiden, und zum anderen dazu verhelfen sollen, zukünftige Kooperationen zu ermöglichen. Ob diese Motive dabei zur Geltung kommen können, hängt von strukturellen Bedingungen der Verteilungssituation ab. Damit das eigene Abgabeverhalten oder auch Hilfeverhalten durch soziale Kontrolle beeinflusst wird, oder auch eine Signalwirkung für potentielle Kooperationspartner erlangen kann, muss es natürlich beobachtbar sein. Welche eigenständigen Effekte dabei unterschiedliche Informationsbedingungen haben können, werden wir im Zusammenhang mit dem strategischen Motiv und der Fairnessnorm thematisieren (Absatz 7-9 in diesem Kapitel).

2 Es lässt sich hierbei nicht überprüfen, ob die Bonuszahlung des Arbeitgebers aus Gründen der Belohnung oder zur Setzung von Anreizen erfolgt. Ein Argument, welches dafür spräche, die Bonuszahlung in dieser Verteilungssituation allein als Belohnung zu interpretieren, ist die Überlegung, dass der Arbeitgeber alle seine Mitarbeiter in gleicher Weise anspornen möchte (vgl. Akerlof / Yellen 1990). Jedoch zeigen Studien, dass spezifisch gesetzte Anreize Wettbewerb erzeugen und somit die Leistung von Mitarbeitern steigern können (vgl. Frey / Meier 2004). Aus dieser Sicht wären unterschiedlich hohe Zahlungen zu erwarten (vgl. Lazear / Rosen 1981). 
Das beobachtbare Abgabeverhalten kann dem Verteilungsmuster unterschiedlicher Fairnessnormen entsprechen (siehe z.B. Hegdtved 1992; Pointner 2012). Da sich die Arbeitnehmer bezüglich ihres Beitrags zum Unternehmenserfolg in Form von Arbeitsleistung und Betriebszugehörigkeit explizit unterscheiden, und dies einen Indikator für den individuellen Verdienst am Unternehmensgewinn darstellt, ist es plausibel, davon auszugehen, dass die Fairnessnorm dem Proportionalitätsprinzip (Adams 1965) folgt. Auch zeigen empirische Studien zu Fairness und Gerechtigkeit, dass eine proportionale Auszahlung nach Kriterien wie Leistung oder Anstrengung im Vergleich zu Gleichverteilungen dominieren, sofern die Bedingungen gegeben sind, diese Unterscheidungen machen zu können (Almas et al. 2010; Cappelen et al. 2007). Demnach erwarten wir Folgendes:

H1.1: Je höher die Arbeitsleistung des Arbeitnehmers, desto höher sein erhaltener Bonus.

H1.2: Je länger die Beschäftigungsdauer des Arbeitnehmers, desto höher sein erhaltener Bonus.

Der oben beschriebene Unterschied zwischen dem DS und dem US führt dazu, dass der Geber im US eine strategische Entscheidung zu fällen hat. Der Geber muss antizipieren, welchen Anteil der Empfänger gerade noch zu akzeptieren bereit ist, und genau diesen abgeben, um seine eigene Auszahlung zu maximieren. Der Unterschied in den durchschnittlichen Abgaben zwischen dem DS mit 28\% (Engel 2011) und dem US mit ca. 40\% (Oosterbeek et al. 2004) ist demnach auf strategische Motive zurückzuführen. In unserer Verteilungssituation haben die Arbeitnehmer keine Möglichkeit mit dem Arbeitgeber über die Bonuszahlung zu verhandeln oder gar ein Veto einzulegen. Dennoch unterscheiden sie sich in ihrer Sanktionsmacht. Demnach kann die Möglichkeit bestehen, dass ein Arbeitgeber aus strategischen Motiven auf ein hohes Sanktionspotential mit einer höheren Zahlung reagiert. Es sind drei Merkmale, die wir als Indikatoren für Sanktionsmacht verwenden. Zum einen kann der Arbeitnehmer Mitglied in einer einflussreichen Gewerkschaft sein. Die Probanden wurden dabei explizit darauf hingewiesen, dass eine Gewerkschaft die Interessen der Arbeitnehmer gegenüber dem Arbeitgeber vertritt, womit das Sanktionspotential der Gewerkschaft salienter gemacht werden sollte. Demnach erwarten wir einen positiven Effekt der Gewerkschaftsmitgliedschaft auf die Bonuszahlung.

H2.1: Die Bonuszahlung ist für jene Arbeitnehmer höher, die ein Mitglied in einer Gewerkschaft sind.

Eine Möglichkeit des Arbeitnehmers selbst zu sanktionieren kann darin bestehen, dass er das Unternehmen zu verlassen droht, wenn er sich ungerecht behandelt fühlt. Dies hängt allerdings von den Arbeitsmarktbedingungen ab. Die Arbeitsmarktchancen können dabei einmal zugunsten des Arbeitnehmers sein, wenn dieser leicht einen beruflichen Posten in einem anderen Unternehmen finden kann und zugleich der Arbeitgeber große Schwierigkeiten dabei hätte, diesen gleichwertig zu ersetzen. Sind die Arbeitsmarktchancen zugunsten des Arbeitgebers, gilt hingegen die umgekehrte Situation. Diese institutionellen Bedingungen bestimmen damit ein Machtverhältnis (Coleman 1991) zwischen beiden Akteuren und ein rationaler Akteur weiß dieses Machtungleichgewicht zu seinem Vorteil zu nutzen.

H2.2: Die Bonuszahlung für Arbeitnehmer mit günstigen Arbeitsmarktchancen ist höher als für Arbeitnehmer mit ungünstigen Arbeitsmarktchancen.

Sanktionsmacht kann des Weiteren vom sozialen Status ausgehen (Wolbring et al. 2013). In diesem Zusammenhang geht mit sozialem Status, gemessen über die Berufsposition (Treiman 1977), eine größere Verantwortung einher. Demnach steigt mit dem Status auch das 
Ansehen des Arbeitnehmers, was ihm tendenziell die Möglichkeit gibt, für die Durchsetzung seiner Interessen mehr Ressourcen mobilisieren zu können.

\section{H2.3: Die Bonuszahlung ist umso höher, je höher der Berufsstatus des Arbeitnehmers ist.}

Motive wie der Erwerb einer vorteilhaften Reputation oder der Effekt sozialer Erwünschtheit können prinzipiell nur zur Geltung kommen, wenn der Geber sich in der Ressourcenallokation beobachtet weiß und sich demnach unter sozialer Kontrolle der Empfänger, bzw. auch unbeteiligter Dritter, welche Informationen über das Verhalten an relevante Andere weitergeben können, befindet (z.B. Nowak / Sigmund 2005 über die Mechanismen der Entstehung indirekter Reziprozität). Die Informationsbedingungen, unter welcher die Allokation stattfindet, können also Motive für die Abgabe hinzufügen bzw. ,ausschalten“. Wir unterscheiden hierbei zwischen zwei Informationsdimensionen, von denen wir erwarten, dass sie die Motive unterschiedlich beeinflussen: Einerseits das Wissen der Arbeitnehmer darüber, dass es einen Bonus zu verteilen gibt, und zum anderen das Wissen der Arbeitnehmer darüber, wie hoch die Bonuszahlung in jeder einzelnen Allokation ausfällt.

Tabelle 1: Informationsbedingungen

\begin{tabular}{ccc}
\hline & $\begin{array}{c}\text { AN kennen die Bo- } \\
\text { nusallokation nicht }\end{array}$ & $\begin{array}{c}\text { AN kennen die } \\
\text { Bonusallokation }\end{array}$ \\
\hline $\begin{array}{c}\text { AN wissen, } \\
\text { dass Bonus möglich ist }\end{array}$ & teiltransparent & transparent \\
\hline $\begin{array}{c}\text { AN wissen nicht, } \\
\text { dass Bonus möglich ist }\end{array}$ & intransparent & \\
\hline
\end{tabular}

Anmerkung: AN steht für Arbeitnehmer.

Die Bonuszahlung kann demnach in unterschiedlichem Ausmaße transparent sein (siehe Tabelle 1). Wir argumentieren, dass in der komplett intransparenten Situation allein die subjektiven Normen des Arbeitgebers über die Höhe der einzelnen Bonuszahlungen entscheiden. Besitzen die Arbeitnehmer Kenntnis darüber, dass die Höhe des Unternehmensgewinns eine Bonuszahlung zulässt, bzw. dass ein Anlass für eine Bonuszahlung gegeben ist, wird der Arbeitgeber damit rechnen, dass diese eine Belohnung erwarten. Eine ausbleibende bzw. allzu geringe Zahlung könnte zu Unzufriedenheit unter den Arbeitnehmern führen, weshalb unter dieser Informationsbedingung ein externer Anreiz für eine Bonuszahlung hinzukommt. Die soziale Kontrolle wird in der komplett transparenten Situation noch einmal gesteigert. Denn neben der Tatsache, dass die Arbeitnehmer ihre Belohnungen miteinander vergleichen können, werden sie sich auch ein generelles Urteil über die Großzügigkeit des Arbeitgebers bilden. Ist die Allokation für alle offen einsehbar, hat das Einfluss auf die Reputation des Arbeitgebers. Sowohl das Erlangen einer positiven Reputation als auch das Vermeiden einer negativen Reputation stellt einen weiteren externen Anreiz für eine Bonuszahlung dar.

H3: Je transparenter die Verteilungssituation ist, umso höher ist die durchschnittliche Bonuszahlung.

Obgleich wir davon ausgehen, dass die Fairnessnorm, welche in diesem Kontext zur Geltung kommt, dem Proportionalitätsprinzip folgt, spielt die Transparenz der Allokation eine Rolle dabei, bis zu welchem Grad dieser Norm gefolgt wird (Cook / Hegtvedt 1983). Hierbei kommt es nur auf eine der Informationsdimensionen an, nämlich das Wissen der Arbeitnehmer darüber, wie viel der Arbeitgeber in den einzelnen Fällen als Bonus gezahlt hat, also 
ob die Bonuszahlung offen anstatt geheim abläuft. Tauschen sich die Arbeitnehmer über den erhaltenen Bonus aus und stellen fest, dass die Auszahlungen sehr ungleich sind, würden sich einige benachteiligt fühlen. Daraus könnten Unzufriedenheit und schlechtere Arbeitsleistung folgen. Allein um dies zu vermeiden, würde der Arbeitgeber entsprechend der Gleichheitsnorm eine eher homogene Verteilung gegenüber einer Auszahlung nach jenen Kriterien vorziehen, die er eventuell bevorzugt. Durch diese soziale Kontrolle, die auf den Arbeitgeber ausgeübt wird, steigt der Druck einer sozialen Erwartung zu entsprechen, für die er eine höhere gesellschaftliche Legitimität vermutet. Hierbei ist es eine rein empirische Frage, ob die Proportionalitätsnorm bzgl. der Leistung oder bzgl. der Zugehörigkeitsdauer stärker abgeschwächt wird.

H4.1: Die postulierten Effekte der Fairnessnorm aus den Hypothesen 1.1 und 1.2 sind in der komplett transparenten Situation schwächer ausgeprägt als in der intransparenten und der teiltransparenten Situation.

Die untersuchten strategischen Motive reagieren dabei auf die andere Informationsdimension, nämlich allein darauf, ob die Arbeitnehmer überhaupt Kenntnis von einer zu verteilenden Ressource haben. In der komplett intransparenten Situation sollte der Proband überhaupt nicht auf das Sanktionspotential der Arbeitnehmer reagieren.

H4.2: Die Effekte der Sanktionsmacht aus den Hypothesen 2.1, 2.2 und 2.3 treten nur in der komplett- und teiltransparenten Situation auf, aber nicht in der intransparenten Situation.

Das faktorielle Design, welches für die Überprüfung der Hypothesen sowohl Within-Variation als auch Between-Variation berücksichtigt, wird im Folgenden beschrieben.

\section{Design und Operationalisierung}

Für die Untersuchung der Frage danach, was Menschen dazu veranlasst, zu geben und zu nehmen, werden meist Laborexperimente durchgeführt. Dabei ist diese Methode nicht unumstritten. Da die Untersuchungen zum großen Teil mit studentischen Stichproben in einer künstlichen Entscheidungsumwelt durchgeführt werden, stellt sich die Frage, was diese Ergebnisse über das menschliche Verhalten in der realen gesellschaftlichen Welt aussagen (z.B. Levitt / List 2007). Zum Zweck eines größeren Realismus und einer größeren Repräsentativität für spezifische Populationen werden einerseits Feldstichproben z.B. einer bestimmten Berufsklasse in das Experimentallabor geholt (z.B. Fehr / List 2002) oder es werden aus Laborexperimenten gewonnene Hypothesen im Feld einer weiteren Prüfung unterzogen (z.B. Gneezy / List 2006 für das im Labor intensiv untersuchte „Gift-Exchange-Game“). Schließlich ist auch zur Überprüfung einiger soziologischer Theorien eine kontrollierte Feldbeobachtung notwendig, da man dem Gegenstand, wie flüchtigen Verhaltensweisen und Interaktionen in spezifischen sozialen Situationen, mit anderen Methoden nicht gerecht werden kann (zur Überprüfungen von Hypothesen zu sozialem Status z.B. Jungbauer-Gans et al. 2005; Wolbring et al. 2013). Jedoch nach Falk und Heckman (2009) sind Feldexperimente nicht per se informativer. Die entscheidende Frage sei die Isolierung des Effekts eines Stimulus unter Kontrolle von Kovariaten wie Personenmerkmalen, institutionellen Bedingungen etc. Diese Erhebungsbedingungen (Setting) und die Zielpopulationen müssten in Feldexperimenten klar spezifiziert sein. Und da das Setting im Labor viel sicherer kontrolliert (aber freilich nicht jedes simuliert) werden könne, spreche dies für das Laborexperiment. Gerade soziologische Erklärungen basieren aber nicht selten auf mehr als ein oder zwei Einflussfaktoren. Würden aber alle in den Hypothesen entwickelten Prädiktoren der Bonuszahlung in dieser Studie systematisch variiert, ergäbe sich in einem Laborexperiment 
schnell eine kaum zu realisierende Anzahl von Untersuchungsgruppen mit jeweils spezifischen Charakteristika.

Eine Methode, welche die Vorzüge des Feldexperiments und des Laborexperiments nutzt, ist der faktorielle Survey (Beck / Opp 2001; Jasso 2006; Rossi / Anderson 1982; Rossi 1979; Wallander 2009). In einem faktoriellen Survey werden fiktive Situationen, Objekte, Personen oder Handlungsalternativen beschrieben (sogenannte Vignetten) und dabei die Ausprägungen (Levels) der jeweils interessierenden unabhängigen Merkmale (Dimensionen) systematisch variiert. Diese fiktiven Situations- oder Personenbeschreibungen werden dann den Probanden zufällig zugeordnet und zur Bewertung vorgelegt. Auf diese Weise ist eine randomisierte Gruppenzuordnung möglich, denn alle Probanden, die dieselbe Vignette beurteilt haben, bilden eine eigene Experimental- bzw. Kontrollgruppe. Damit können einerseits Probanden in eine Situation versetzt werden, die einem bestimmen institutionellen Feld entspricht (siehe z.B. Abraham / Meyer 2012; Barrera / Buskens 2007; Raub / Buskens 2005). Andererseits lassen sich spezifische Feldstichproben rekrutieren, da die Probanden weder zu einem bestimmten Zeitpunkt in ein Labor geholt werden noch im Feld beobachtet werden müssen, sondern auf den in Surveystudien üblichem Wege kontaktiert werden, z.B. via Email. Wir nutzen diese Methode, um die Verteilungssituation einer Bonuszahlung zu analysieren, die in der realen Welt stattfindet, dabei aber nur sehr schwer unter kontrollierten Bedingungen beobachtet werden kann. Zudem erlaubt der faktorielle Survey die Berücksichtigung einer Vielzahl verschiedener unabhängiger Merkmale. Dem schnell wachsenden Umfang möglicher Merkmalskombinationen, der sich aus der Zahl der variierten Dimensionen und ihrer Levels ergibt, wird mit zwei Strategien begegnet. Erstens wird aus der Gesamtheit aller Vignetten, dem sogenannten Vignettenuniversum, eine Stichprobe gezogen, die das Vignettenuniversum repräsentiert. Nur diese Vignetten gehen in die Analyse ein. Zweitens werden mehrere Vignetten zu Decks zusammengefasst (meist 8-12), die den Probanden vorgelegt werden.

Im Rahmen eines faktoriellen Surveys sind also sowohl die Variation mehrerer Mitarbeiter- und Situationsmerkmale als auch die Beobachtung einer fiktiven Bonuszahlung und die Randomisierung der Probanden über die Situationen möglich. Das Design weist demnach alle Merkmale eines echten Experiments auf. Die Probanden müssen dabei nicht mit realen unabhängigen Variablen konfrontiert werden und auch nicht tatsächlich einen Bonus auszahlen. Es ist aufgrund der Randomisierung auch keine komplexe Ziehung einer ( $\mathrm{Zu}-$ falls-)Stichprobe notwendig und wenige Probanden sind ausreichend. Gleichwohl ist die Bestimmung der isolierten, relativen Nettoeffekte der variierten Dimensionen möglich. Darüber hinaus erlaubt die komplexe Beschreibung der Situation und der Personen die Simulation einer realistischen Entscheidungssituation, in der die Befragten die Bedeutung verschiedener Merkmale für ihre Bonuszahlungen abwägen müssen.

Neben Problemen der internen Validität wie der Gefahr der Konstruktion unrealistischer Fälle (Auspurg et al. 2010), Lern- und Ermüdungseffekten (Sauer et al. 2011), Reihenfolgeneffekten (Auspurg / Jäckle 2012), Variationseffekten (Auspurg et al. 2009), Effekten der Formulierung (Eifler / Petzold 2014) oder Verzerrungen durch soziale Erwünschtheit (Eifler 2007, 2010) richtet sich die Hauptkritik am faktoriellen Survey jedoch gegen die Tatsache, dass nur fiktive Urteile bzw. Handlungsintentionen im Hinblick auf fiktive Vignetten berichtet werden (vgl. Lucas 2003). Bisher vorliegende Validierungsstudien am Beispiel abweichenden Verhaltens (Groß / Börensen 2009) oder zu Umzugsentscheidungen (Nisic / Auspurg 2009) zeigen jedoch, dass im Wesentlichen realitätsbezogene Entscheidungen getroffen werden, bei denen das Ausmaß der experimentellen Effekte zwar nicht denen der realen Beobachtungen entspricht, wohl aber ihrer Richtung und relativen Einflussstärke. Aufgrund der genannten Vorteile des FS wird diese Methode neben der Messung von Normen und 
Einstellungen (z.B. Rossi 1979; Rossi / Anderson 1982; Beck / Opp 2001; Mäs et al. 2005; vgl. Wallander 2009) auch angewandt, um Urteile zur Gerechtigkeit von z.B. Löhnen und Steuerabgaben zu messen (z.B. Alves / Rossi 1978; Liebig / Mau 2005; Jasso 2006; Gückelhorn et al. 2014). Dabei lässt sich wie in unserem Design feststellen, ob z.B. etwa eher nach einem Leistungs- oder einem Bedarfsprinzip geurteilt wird und ob es Diskriminierungen gibt. Inhaltlich stehen diese Arbeiten teilweise in engem Zusammenhang zu unserer Untersuchung. Da unsere Arbeit an die vorwiegend labor- und feldexperimentellen Studien zu pro-sozialem Verhalten anknüpft, ergibt sich aber ein konzeptueller Unterschied zu diesen Gerechtigkeitsstudien. Zum einen messen wir keine Urteile, sondern Verhaltensabsichten. Damit zusammenhängend befinden sich zum anderen die Probanden selbst in einer aktiven Rolle, nämlich in der des Gebers. Die Nützlichkeit des FS für diese Form der handlungstheoretischen und auch spieltheoretischen Fragestellungen ist dabei schon mehrfach gezeigt worden (z.B. Barrera / Buskens 2007; Buskens / Weesie 2000; Rooks et al. 2000; Vieth 2004; Abraham / Meyer 2012).

Die Erfassung der Bedingungen einer Bonuszahlung von Arbeitgebern basiert in dieser Studie demnach auf ,als-ob-Annahmen“. Da die Teilnehmer unserer Untersuchung keine reale Auszahlung erhalten und deren Entscheidungen damit konsequenzenlos sind, muss man damit rechnen, dass die Höhe der Abgaben möglicherweise überschätzt wird (vgl. BenNer et al. 2005). Zwar wird in Laborexperimenten regelmäßig um echte Geldbeträge gespielt, die den Teilnehmern nachher auch tatsächlich zustehen, und es ist grundsätzlich denkbar, dass auch im Rahmen eines faktoriellen Surveys echte Geldbeträge ausgezahlt werden. Vor dem Hintergrund der Zielsetzung dieser Untersuchung zugunsten einer höheren externen Validität, Arbeitgeber aus ausgewählten Teilen der Bundesrepublik als Probanden hinzuzuziehen, war diese Option allerdings nicht praktikabel. Folgende Merkmale (Dimensionen) wurden in den Vignetten variiert (siehe Tabelle 2). Es ist bei der Auswahl und der Anzahl der Dimensionen darauf geachtet worden, den Probanden weder zuviel noch zu wenig Information vorzulegen und gleichzeitig die zentralen und theoretisch relevanten Prädiktoren zu erfassen.

\section{Tabelle 2: Dimensionen und Levels}

\begin{tabular}{|c|c|c|c|}
\hline $\begin{array}{l}\text { Beschifftigungsdauer } \\
\text { Leistung } \\
\text { Gewerkschaftsmitglied } \\
\text { Berufsposition } \\
\text { Arbeitsmarktchancen } \\
\text { Alter } \\
\text { Geschlecht }\end{array}$ & $\begin{array}{l}1 \text { Jahr } \\
\text { unter- } \\
\text { Ja } \\
\text { Facharbeiter } \\
\text { zugansten Arbeitnehmer } \\
\text { 30 Jahre } \\
\text { männlich }\end{array}$ & $\begin{array}{l}5 \text { Jahre } \\
\text { durchschnittlich } \\
\text { Nein } \\
\text { Meister } \\
\text { zugunsten Arbeitgeber } \\
45 \text { Jahre } \\
\text { weiblich }\end{array}$ & $\begin{array}{l}10 \text { Jahre } \\
\text { uber- } \\
\text { leitender Angestellter } \\
60 \text { Jahre }\end{array}$ \\
\hline $\begin{array}{l}\text { konstante Merkmale: } \\
\text { unerwartet hoher Unternehmensgewinn } \\
\text { Arbeitnehmer weiB nicht, wieviel er maximal } \\
\text { an Bonus whalten kann }\end{array}$ & & & \\
\hline
\end{tabular}

Das kartesische Produkt der Dimensionen und Levels ergibt ein Vignettenuniversum von 648 möglichen Kombinationen. Hieraus wurde eine d-effiziente Stichprobe von 108 Vignetten gezogen, die eine maximale Unkorreliertheit der Dimensionen (Orthogonalität) bei einer maximalen Varianz der Levels (Level Balance) aufweist (Steiner / Atzmüller 2006; Dülmer 2007; Kuhfeld 2010). Die Vignetten wurden weiterhin auf neun Decks mit je zwölf Vignetten aufgeteilt, sodass jeder Proband die Bonushöhe für zwölf fiktive Personen bestimmen konnte.

Eine wesentliche Erweiterung des faktoriellen Surveys in unserer Studie ergibt sich durch eine weitere kontrollierte Variation der Informationsbedingungen im Hinblick auf die fiktiven Mitarbeiter. Vor der Präsentation der Vignetten wurden die Probanden einer der drei oben erörterten Entscheidungssituationen zufällig zugeordnet. Der erwartete Effekt der Ent- 
scheidungsumwelten liegt darin, dass sie die verschiedenen Motive unterschiedlich begünstigen bzw. hemmen. Einmal sind die Arbeitnehmer darüber informiert, dass es Anlass für eine Bonuszahlung gibt, einmal nicht. Die zweite Bedingung bezieht sich darauf, ob die Bonuszahlung offen oder geheim abläuft. Die Probanden haben keine Kenntnis davon erlangt, dass es mehrere Entscheidungsumwelten gibt.

Eine Bonuszahlung wird daher in dieser Studie sowohl durch die Merkmale der fiktiven Mitarbeiter (Vignette) als auch durch die Merkmale der Situation beeinflusst, in die der Proband versetzt worden ist. Im Experiment werden demnach ein Within- und ein Between-Design kombiniert. Zwei Argumente sprechen für ein solches Vorgehen. Zum einen gehen wir davon aus, dass eine konstante Entscheidungsumwelt für die Probanden eine realistischere Situation darstellt. Zum anderen bestünde durch eine Variation der Informationsbedingungen innerhalb der durch die Probanden zu bewertenden Decks die Gefahr einer kognitiven Überforderung, da wechselnde Informationsbedingungen zur Folge haben, dass womöglich alle Vignettendimensionen anders beurteilt werden müssen.

Die in den Vignetten variierten Personenmerkmale in Kombination mit den ,,vorgeschalteten“"Entscheidungsumwelten ergeben eine experimentell hergestellte Mehrebenenstruktur. In diesem Sinne sprechen wir auch von einem „Multilevel Factorial Survey Design“.

Abbildung 1 zeigt eine Beispielvignette. Neben Informationen zur Entscheidungsumwelt sind die Arbeitnehmer beschrieben. Die fiktive Auszahlung erfolgte über eine 11-stufige Skala von 0 Euro bis 10.000 Euro in 1.000 Euro-Schritten, wobei kein Wert vorgegeben wurde. Außerdem wurden die Probanden darauf hingewiesen, dass sie jede Vignette als einzelnen Fall und demnach unabhängig von den anderen Vignetten beurteilen sollten.

Abbildung 1: Beispielvignette

Hier sind noch einmal die wichtigsten Punkte der Ausgangssituation zusammengefasst:

- Das Unternehmen existiert seit zehn Jahren

- Großer wirtschaftlicher Erfolg in den letzten beiden Jahren

- Die MitarbeiterInnen sind über die wirtschaftliche Situation informiert

- Die Bonuszahlung erfolgt geheim

- Die MitarbeiterInnen wissen nicht, wie viel sie maximal an Bonus bekommen können

- Sie haben selbst noch keinen Bonus erhalten

Fall 7:

Der 45 Jahre alte Mitarbeiter ist seit 10 Jahren bei Ihnen als Meister beschäftigt.

Die Leistung des Mitarbeiters ist überdurchschnittlich. Er ist Mitglied in einer einflussreichen Gewerkschaft.

Die Arbeitskraft hätte keinerlei Schwierigkeiten, eine gleiche Position außerhalb Ihres Unternehmens zu finden. Hingegen

wären die Möglichkeiten für Sie, die Arbeitskraft gleichwertig ersetzen zu können, schlecht.

Welchen Betrag zahlen Sie dieser Person aus? Der Restbetrag geht in Ihr eigenes Vermögen über. (Bitte unter die Leiste klicken)

\begin{tabular}{|c|c|c|c|c|c|c|c|c|c|c|}
\hline $0 €$ & $1.000 €$ & $2.000 €$ & $3.000 €$ & $4.000 €$ & $5.000 €$ & $6.000 €$ & $7.000 €$ & $8.000 €$ & $9.000 €$ & $10.000 €$ \\
\hline$\Gamma$ & $T$ & $T$ & $T$ & $T$ & $T$ & $T$ & $T$ & $T$ & $T$ & I \\
\hline
\end{tabular}

Das Design wurde aufgrund einiger Vorteile hinsichtlich der Rekrutierung und der Darstellbarkeit in einen Online-Survey implementiert (vgl. Berger et al. 2009). Hier ist die Rando- 
misierung der Decks über die Probanden, und damit eines der Kernprinzipien des Experiments, ohne weiteres möglich. Außerdem konnte die Position der Vignetten innerhalb der Decks randomisiert werden, um auf diese Weise Reihenfolgeneffekte zu neutralisieren. Neben der besseren Übersichtlichkeit für die Probanden erlaubte die Online-Implementierung ein Zurückblättern, was Zensierungseffekte vermeiden kann (Auspurg et al. 2009).

Die Rekrutierung der Probanden erfolgte dann ebenfalls online. Die Unternehmer wurden über ihre E-Mailadressen kontaktiert, die über die Handelskammerinformationen und das Verzeichnis „Gelbe Seiten“ recherchiert worden waren. Bei der Auswahl wurde versucht, Unternehmen aus den Bereichen Industrie, Handwerk und Dienstleistung möglichst ausgewogen zu erfassen. Zudem begründet sich diese Form der Rekrutierung in der Möglichkeit, die schwer erreichbare Gruppe der kleinen und mittelständischen Unternehmen für die Studie zu gewinnen. Geschäftsführer kleinerer und mittlerer Unternehmen können sich vermutlich besser in die fiktive Situation hineinversetzen, dass einzelne Mitarbeiter direkt am Unternehmenserfolg beteiligt sind. Außerdem besteht in solchen Unternehmen häufig auch ein direkter Kontakt zwischen dem Arbeitgeber und Arbeitnehmer.

Insgesamt sind 1392 Unternehmer aus den Regionen Hannover, Köln, Leipzig und Teilen Bayerns angeschrieben worden, von denen 171 die Startseite des Survey-Experiments öffneten. 81 Probanden haben insgesamt 861 Bonuszahlungen vorgenommen, die als Analysegrundlage dienen. Allerdings haben nur 73 Probanden die Befragung beendet, da am Ende der Befragung noch Merkmale zur Person und zum Unternehmen abgefragt wurden, was weitere Abbrüche bedingte. ${ }^{3}$ Tabelle 3 enthält neben den sozio-demographischen und den unternehmensbezogenen Merkmalen des gesamten Samples zusätzlich diese Angaben nach den situativen Informationsbedingungen getrennt, um systematische Unterschiede im Between-Design bzw. nicht perfekte Randomisierungen aufzudecken.

Tabelle 3: Probanden- und Unternehmensmerkmale in Experimentalgruppen

\begin{tabular}{lcccc}
\hline Merkmal & $\begin{array}{c}\text { Durchschnitt/Anteil } \\
\text { insgesamt }\end{array}$ & intransparent & teiltransparent & transparent \\
\hline Personalverantwortung & $100 \%$ & $100 \%$ & $100 \%$ & $100 \%$ \\
Alter & 51 & 49 & 53 & 51 \\
$\begin{array}{l}\text { Im Unternehmen } \\
\text { seit ... Jahren }\end{array}$ & 15 & 17 & 13 & 16 \\
$\begin{array}{l}\text { Unternehmen existiert } \\
\text { seit ... Jahren }\end{array}$ & 32 & & & \\
Unternehmensgrö6e & 37 & 53 & 45 & 29 \\
$\begin{array}{l}\text { Dienstleistung } \\
\text { Industrie }\end{array}$ & $46 \%$ & $45 \%$ & 23 & 32 \\
Handwerk & $31 \%$ & $30 \%$ & $33 \%$ & $41 \%$ \\
\hline
\end{tabular}

3 Die in der Unternehmensforschung allgemein geringen Rücklaufquoten werden in der Regel mit einem grundlegenden Misstrauen gegenüber der Informationsweitergabe generell begründet, was sich auch in den Abbrüchen bei Fragen zum Unternehmen widerspiegelt. Zudem wird häufig auf mangelnde Zeit gerade in kleinen und mittleren Unternehmen verwiesen. Schließlich nehmen die Anfragen an Unternehmen zu Online-Umfragen immer mehr zu, sodass die Bereitschaft insgesamt sinkt. 
Die Tatsache, dass alle Probanden auch Führungsverantwortung im Unternehmen tragen, lässt eine akzeptable externe Validität vermuten. ${ }^{4}$ Mit Mittelwerten zwischen 49 und 53 Jahren bei einem Gesamtmittelwert von 51 Jahren ist die Streuung im Alter zwischen den Situationen sehr gering. Auch die Beschäftigungsdauer im Unternehmen variiert zwischen durchschnittlich 13 und 17 Jahren bei einem Gesamtmittelwert von 15 Jahren nur gering. Ein etwas deutlicherer Unterschied zeichnet sich im Unternehmensalter ab, das im Mittel zwischen 23 Jahre in der intransparenten Situation und 45 Jahren in der teiltransparenten erkennbar schwankt. Der mittlere Wert im gesamten Sample beträgt 32 Jahre. Auch wenn die Variation in der Unternehmensgröße mit Mittelwerten zwischen 53 Mitarbeitern in der intransparenten Situation und 26 Mitarbeitern in der teiltransparenten Situation auf den ersten Blick groß erscheint, so wird hier deutlich, dass vor allem kleine Unternehmen an der Studie teilgenommen haben. Am häufigsten beteiligten sich Unternehmen aus der Dienstleistungsbranche, gefolgt von Unternehmen aus der Industrie und schließlich dem Handwerk. Diese relativen Häufigkeiten sind auch in den unterschiedlichen Situationen gegeben, allerdings sind in der teiltransparenten Situation nur etwa halb so viele Handwerksbetriebe repräsentiert wie in den beiden anderen Situationen. Die Verteilung der Probanden- und Unternehmensmerkmale legt insgesamt eine weitestgehend gelungene Randomisierung der Probanden über die Situationen im Between-Design nahe.

\section{Ergebnisse}

Die Verteilung der Bonuszahlungen in unserer Studie mit durchschnittlich 42,7\% liegt höher als die Verteilung des „Kuchens“ in den meisten Diktator-Spielen mit einer durchschnittlichen Abgabe von 28\% (Engel 2011) und ähnelt eher den Ergebnissen aus Ultimatum-Spielen mit durchschnittlich 40\%. Mögliche Ursachen dafür werden im Diskussionsteil aufgegriffen (Kap. 5, Abs. 4).

Abbildung 2: Mittlere Bonushöhe mit 95\%-Konfidenzintervall in unterschiedlichen Informationsbedingungen

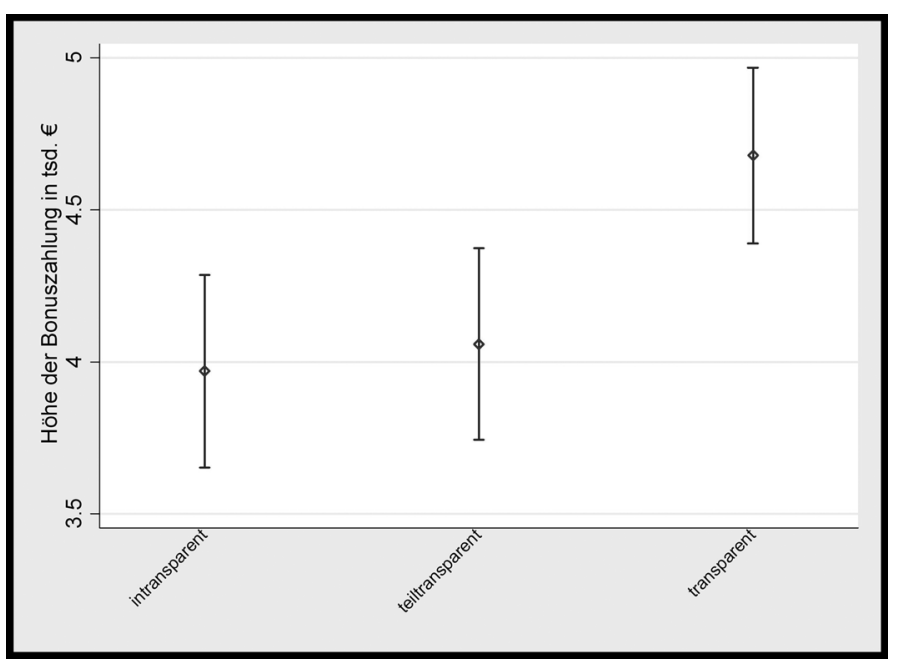

4 Ein Pretest unter Studierenden ergab im Vergleich zu den hier präsentierten Ergebnissen mit der Feldstichprobe eine deutlich höhere durchschnittliche Auszahlung, was darauf zurückgeführt werden kann, dass es sich um eine für die Entscheidungssituation zu unspezifische Stichprobe handelte. 
Dabei unterscheiden sich die mittleren Bonuszahlungen zwischen den Situationen, und zwar wie in $\mathrm{H} 3$ behauptet. In Abbildung 2 lässt sich erkennen, dass sich der Wert in der komplett transparenten Situation von 4.689 Euro auf dem 5\%-Signifikanzniveau von den Werten der intransparenten Situation von 3.969 Euro $(\mathrm{t}=3.24, \mathrm{p}=0.0006)$ und der teiltransparenten Situation von 4.059 Euro $(t=2.84, p=0.0023)$ unterscheidet. Die Differenz zwischen der intransparenten und der teiltransparenten Situation ist allerdings sehr gering und demnach auch nicht statistisch signifikant $(\mathrm{t}=0.4, \mathrm{p}=0.3469)$. Dennoch ist erkennbar, dass mit jeder weiteren Informationsdimension die hinzugeschaltet wird, die Bonuszahlung steigt, indem, wie wir es begründen, stets weitere Motive zugunsten einer höheren Abgabe zur Geltung kommen.

Zur Überprüfung der restlichen Hypothesen wurden zwei Regressionsmodelle gerechnet: Jeweils ein Random-Effects-Modell mit geclusterten Standardfehlern (Rabe-Hesketh / Skrondal 2008) einmal für das Hauptmodell ohne die situationsspezifische Effekte und eins mit situationsspezifischen Effekten (Regressionstabellen im Anhang, Tabellen 4 und 5). ${ }^{5}$ In Abbildung 3, worin nicht nach den Situationen unterschieden wird, zeigen jene Arbeitnehmermerkmale den stärksten Effekt, welche wir in H1.1 und H1.2 mit dem Fairnessmotiv erklären, nämlich die Arbeitsleistung und die Beschäftigungsdauer. Wie erwartet tritt ein zunehmender Effekt mit aufsteigender Wertigkeit auf den Merkmalen ein. Insgesamt ist der Effekt der Arbeitsleistung stärker als jener der Beschäftigungsdauer. So bekommt ein Arbeitnehmer mit überdurchschnittlicher Arbeitsleistung durchschnittlich 2.187 Euro mehr als einer mit unterdurchschnittlicher Leistung. Und die Bonusdifferenz zwischen zehnjähriger und einjähriger Dauer beträgt 1.579 Euro. Jedoch ist der Effekt der mittleren Ausprägung der Zugehörigkeitsdauer stärker als jener der Leistung. Die Indikatoren für Sanktionsmacht zeigen nur teilweise einen Effekt. Gewerkschaftsmitgliedschaft und die Arbeitsmarktchance haben in diesem Modell keinen Einfluss auf die Höhe der Bonuszahlung. Demnach können H2.1 und H2.2 für das Gesamtmodell nicht belegt werden. Und obgleich der Arbeitgeber in seiner Belohnung nach Berufsposition unterscheidet, trifft unsere Hypothese H2.3 insofern nicht zu, als für die mittlere Kategorie der Meister der größere Bonusvorteil gegenüber den Facharbeitern vorliegt als für die leitenden Angestellten.

5 Neben dem berichteten RE-Modell ist zusätzlich ein Modell mit den Probanden- und Unternehmensmerkmalen geschätzt worden. Darüber hinaus wurde ein Fixed Effects-Modell berechnet. In beiden Fällen zeigen sich keine substanziellen Unterschiede, sodass sich die Koeffizienten als robust erweisen, was sich auch im Hausman-Test bestätigt. Um möglichst viel statistische Power bei gleichzeitig möglichst guter Effizient zu erhalten, wird daher hier nur das RE-Modell ohne Probanden- und Unternehmensmerkmalen dokumentiert. Ebenfalls aus Fallzahlgründen werden auch Probanden berücksichtigt, die nicht alle Bonuszahlungen vorgenommen haben (vgl. Tabelle zu den Missings in den Bonuszahlungen im Anhang, Tabelle 6). 
Abbildung 3: Regressionseffekte der Vignettendimensionen mit 95\%-Konfidenzintervall, nicht unterschieden nach Informationsbedingungen.

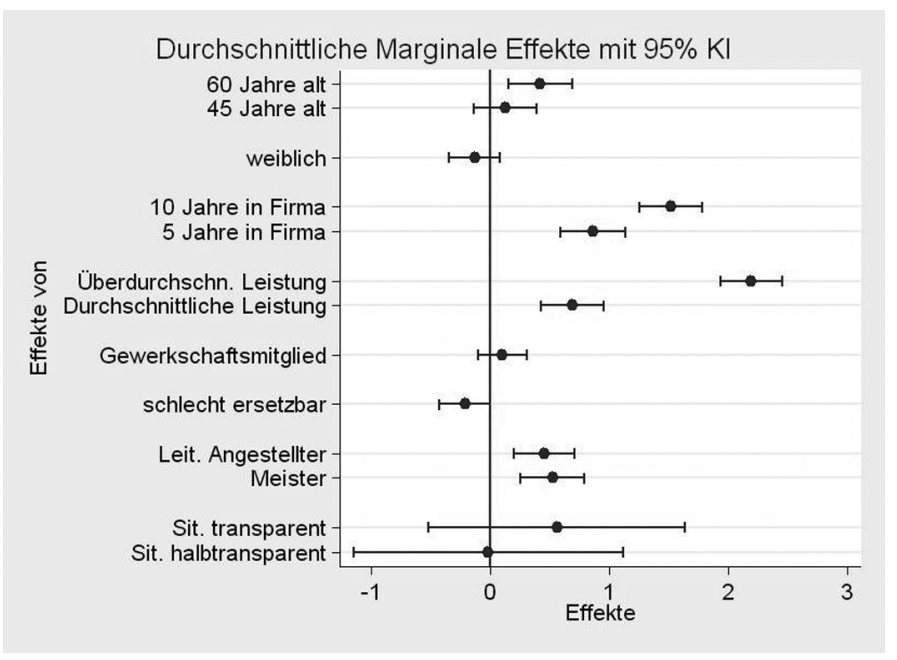

Die eigentlich interessanten Effekte sind dabei die Interaktionen dieser Arbeitnehmermerkmale mit den unterschiedlichen Informationsbedingungen. In Hypothese 4.1 wird behauptet, dass die Effekte der Fairnessnorm in der komplett transparenten Situation im Vergleich zu den beiden anderen Entscheidungsumwelten geringer sind. Tatsächlich schwächen sich die Effekte aller Ausprägungen von der Beschäftigungsdauer und der Arbeitsleistung teilweise deutlich ab (Abbildung: 4).

Abbildung 4: Regressionseffekte für Arbeitsleistung (Referenz: unterdurchschnittlich) und Beschäftigungsdauer (Referenz: $1 \mathrm{Jahr}$ ) in unterschiedlichen Informationsbedingungen

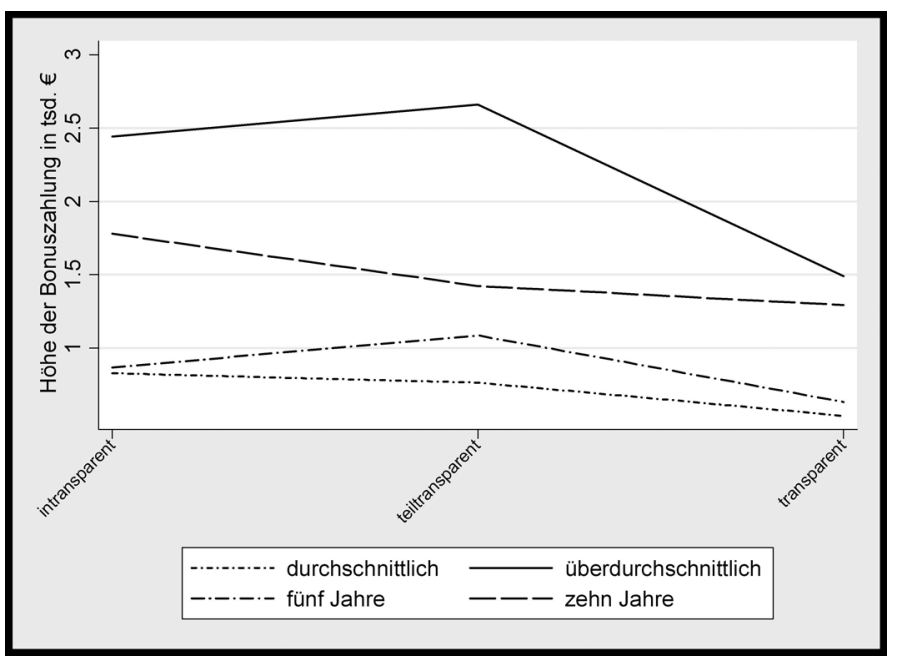

Der einzig signifikante Rückgang gilt jedoch nur für überdurchschnittliche Arbeitsleistung, welcher in der komplett transparenten Situation um 1.160 Euro geringer ist als im Vergleich 
zu den beiden anderen Situationen. Vergleicht man dies mit dem Rückgang des Effekts zehnjähriger Beschäftigungsdauer von etwa 350 Euro, so zeigt sich hier, dass die Divergenz zwischen einer subjektiv fairen Teilung und jener, die vermuteten sozialen Erwartungen gerecht zu werden versucht, sich zwischen Zugehörigkeitsdauer und Leistung drastisch unterscheiden. Dabei ist interessant, dass sich die teils deutlich unterschiedlich starken Effekte von Arbeitsleistung und Betriebszugehörigkeit in der komplett transparenten Situation fast angleichen, wobei sie sich unter Teiltransparenz noch um 1.400 Euro unterscheiden. Die Effekte aus H1.1 und H1.2 bleiben aber in allen Situationen beibehalten.

Das strategische Motiv sollte nach H4.2 allein in den komplett- und teiltransparenten Situationen nachweisbar sein. Zwar ist in der komplett intransparenten Situation kein einziger Effekt der Sanktionspotentiale, so wie es behauptet wurde, vorhanden (Abbildung 5), allerdings tritt auch in der komplett transparenten Situation nur ein einziger signifikanter Effekt auf, nämlich dass einzig die Berufsposition des Meisters im Vergleich zum Facharbeiter einen Unterschied macht. Allein in der teiltransparenten Situation treten die erwarteten Unterschiede in der Höhe der Bonuszahlung für diese Merkmale ein.

Abbildung 5: Regressionseffekte für Gewerkschaftsmitgliedschaft, Arbeitsmarktchance (Referenz: zugunsten des Arbeitgebers) und Berufsposition (Referenz: Facharbeiter) in unterschiedlichen Informationsbedingungen

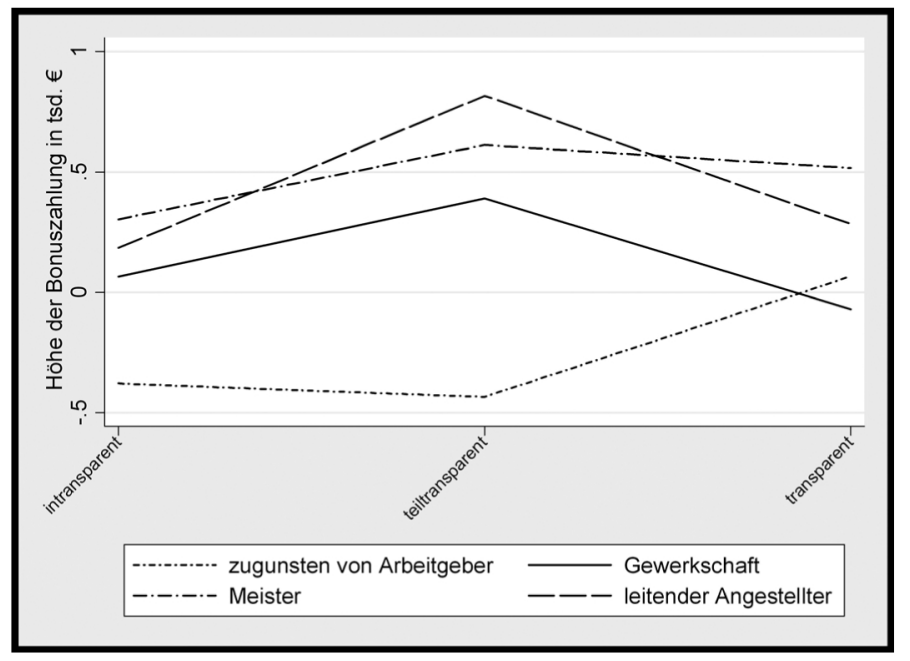

Demnach können wir zwar nachweisen, dass strategische Motive allein zur Geltung kommen, wenn die potentiellen Empfänger auch Erwartungen über eine Belohnung bilden können und der Geber auch davon weiß. Kommt allerdings die andere Informationsdimension noch hinzu, nämlich das Wissen der potentiellen Empfänger darüber, wie viel im Einzelnen ausgezahlt wird, so gehen die Effekte der Strategiemotive genau wie die der Fairnessmotive zurück. Die Effekte der Interaktionsterme zwischen den Strategiemotiven und den Informationsbedingungen werden hier nicht statistisch signifikant. Die Ergebnisse stützen die Hypothese 4.2 demnach nicht. 


\section{Diskussion}

Ziel dieser Arbeit ist es, mittels eines faktoriellen Surveys zu prüfen, welchen relativen Effekt strategische Motive als Reaktion auf Sanktionspotential und Fairnessmotive in einer Verteilungssituation ausüben. Da beide Motive im Wesentlichen durch die Transparenz der Verteilungssituation bestimmt werden, sind die Probanden zufällig in eine von drei Entscheidungsumwelten zugeordnet worden, die sich in den Informationsbedingungen unterscheiden. Damit kann zusätzlich geprüft werden, ob sowohl das Vorhandensein als auch der Grad der Ausprägung beider Motive durch soziale Kontrolle bestimmt wird. Die Untersuchung erfolgt am Beispiel einer Bonuszahlung in einem Unternehmen. Der Arbeitgeber entscheidet dabei, wie eine fiktive Summe von 10.000 Euro zwischen sich und einem Arbeitnehmer aufgeteilt wird.

Zunächst gilt, dass die Wirkung aller Merkmale, die einen relevanten Effekt auf die Bonuszahlung haben, von der Entscheidungsumwelt abhängt. Am deutlichsten wird dies am Beispiel der Bevorzugung von Arbeitnehmern mit höherer Arbeitsleistung. Dieses Merkmal führt in allen Entscheidungsumwelten zu den stärksten Unterschieden in der Bonuszahlung, jedoch bewirkt die Offenheit der Zahlung eine starke Abschwächung. Die Differenz zwischen den Effekten in der intransparenten Situation und der komplett transparenten Situation zeigt den Einfluss wahrgenommener Erwünschtheit und macht damit den Unterschied zwischen subjektiven und mutmaßlich sozial erwünschten Fairnessnormen deutlich. Dass sich im Vergleich zur Arbeitsleistung der Effekt zehnjähriger Beschäftigungsdauer zwischen den Entscheidungsumwelten deutlich weniger unterscheidet, lässt den Schluss zu, dass diese Divergenz bezüglich der Leistung eindeutig größer ist, als bezüglich der Zugehörigkeitsdauer. Die Ergebnisse weisen darauf hin, dass in zukünftigen Analysen zu Fairnessnormen die genauen Informationsbedingungen einer Verteilungssituation explizit berücksichtigt werden sollten.

Die Effekte des Sanktionspotentials sind hingegen weniger eindeutig. Den Statuseffekt haben wir mit dem damit variierenden Sanktionspotential begründet. Diese Effekte lassen sich aber allein damit nicht konsistent interpretieren, da das Belohnungsmuster nicht konstant bleibt. Dies lässt vermuten, dass von den Berufskategorien des Meisters und des leitenden Angestellten unterschiedliche Effekte ausgehen. Der Arbeitgeber fürchtet das Sanktionspotential des leitenden Angestellten, aber nur in der teiltransparenten Situation. Und der Meister erhält seine Bevorzugung aufgrund einer Würdigung seiner Arbeit, und das im Grunde unabhängig von der Entscheidungsumwelt. Zugespitzt formuliert: Der Arbeitgeber würdigt den Meister und fürchtet den leitenden Angestellten. Inwiefern dies von der Stichprobenzusammensetzung verursacht ist, lässt sich dabei nicht zuverlässig untersuchen, da die Angaben der Probanden zu sämtlichen soziodemographischen Merkmalen sehr unvollständig sind. Die Klärung dieser Frage würde demnach weitere Untersuchungen benötigen. Insgesamt lässt sich sagen, dass zum einen die Fairnessmotive einen deutlich stärkeren Einfluss auf die Bonuszahlung ausüben als die Strategiemotive. Zum anderen zeigt sich, dass die Wirkung der sozialen Kontrolle in der komplett transparenten Entscheidungssituation die Strategiemotive ,ausschaltet“. Dies ist interessant, weil unter dieser Bedingung offenbar nicht nur soziale Erwünschtheitseffekte von den Arbeitnehmern auf den Arbeitgeber ausgehen, sondern die Arbeitnehmer sich auch gegenseitig unter sozialer Kontrolle befinden können, was insbesondere deren strategischen Handlungsspielraum beeinträchtigt und der Arbeitgeber dies vermutlich antizipiert. Erklärungsbedürftig ist schließlich auch die insgesamt hohe Abgabe von etwa 43\% und insbesondere jene in der intransparenten Situation mit knapp 40\%. Gerade unter dieser Informationsbedingung, welche jegliche soziale Kontrolle der Arbeitnehmer auszuschalten beabsichtigt, wäre eine deutlich geringere Zahlung zu erwarten gewesen. 
Vier mögliche Erklärungen dafür scheinen plausibel, dass dies nicht eingetreten ist: i) Trotz der Intransparenz der Verteilungssituation für die Arbeitnehmer ist die Erhebungssituation freilich nicht anonym. Demnach muss man damit rechnen, dass der Proband auch in dieser Situation auf soziale Erwünschtheit reagiert, die er von Seiten der Forscher vermutet (siehe z.B. Krumpal 2013). ii) Eine entscheidende Grundregel für verhaltensökonomische Laborexperimente konnte in dieser Untersuchung nicht erfüllt werden, nämlich die Anreizkompatibilität über die Bezahlung der Probanden in Abhängigkeit ihrer Entscheidungen im Experiment (Friedman et al. 2009). Durch das Fehlen einer reellen Auszahlung können materielle Eigeninteressen der Probanden nicht hinreichend salient gemacht werden, weshalb faires Verhalten in diesem Design gewissermaßen kostenlos ist und damit nach oben verzerrt sein wird. Andererseits könnte man inhaltliche Argumente anbringen: iii) Zum einen möchte der Arbeitgeber seine Arbeitnehmer motivieren und zahlt ihnen deshalb so viel. Unser Design erlaubt es allerdings nicht zwischen den Motiven des Anreizes und dem der Belohnung zu unterscheiden. iv) Zum anderen kann es aber auch sein, dass der Arbeitgeber keinen alleinigen Besitzanspruch an die Ressource gegenüber sich selbst moralisch rechtfertigen kann, da die Arbeitnehmer an deren Produktion beteiligt sind. Letztere Vermutung ließe sich dabei mit einem laborexperimentellen Design prüfen. Bezüglich all dieser Erklärungen ist aber noch einmal zu berücksichtigen, dass die Arbeitnehmer in allen Situationen keine Kenntnis davon haben, wie viel sie maximal bekommen können. Variiert man diesen Faktor für die teil-und komplett transparente Situation noch zusätzlich, wäre bei Kenntnis der Arbeitnehmer von der Größe des Kuchens eine noch höhere Bonuszahlung zu erwarten.

Darüber hinaus sind die Ergebnisse mit Verweis auf die eingesetzte Methode und die Stichprobe auch einigen Einschränkungen unterworfen. So wurden im Gegensatz zu Laborund Feldexperimenten in unserer Untersuchung keine realen Handlungen beobachtet. Wie im Kapitel „Design“ bereits erwähnt, werden mit dem FS neben Normen ausschließlich Verhaltensintentionen auf der Basis fiktiver Entscheidungssituationen gemessen, die keine validen Indikatoren für tatsächliches Verhalten darstellen müssen. Darüber hinaus kann die Höhe der Bonuszahlungen auch durch die Vignettenkonstruktion, also die Beschreibung der fiktiven Mitarbeiter verzerrt worden sein. Zwar sind keine unplausiblen Fälle im Design möglich gewesen, und möglichen Ermüdungs-, Lern- oder Reihenfolgeeffekten ist mit einer Randomisierung der Vignettenposition in den Decks begegnet worden; Positionseffekte der variierten Dimensionen innerhalb der Vignettenbeschreibungen sind allerdings nicht ausgeschlossen. Zudem ist es möglich, dass die variierten Treatments den postulierten Mechanismus der Sanktionsmacht nur unzureichend abbilden. Alternative Operationalisierungen wie z.B. über Betriebsräte oder Gleichstellungsbeauftragte könnten hierbei Aufschluss geben. In dieser Untersuchung sind die Informationsbedingungen der Präsentation der fiktiven Mitarbeiter experimentell vorangestellt worden. Das entspricht technisch einer Variation auf der Ebene der Decks. Dieses Vorgehen wurde vor allem mit einer größeren Realitätsnähe und der Gefahr der kognitiven Überforderung der Probanden begründet. Gleichwohl wäre es interessant zu wissen, ob eine Variation innerhalb der Decks auf der Ebene der Vignetten zu vergleichbaren Ergebnissen kommt. Schließlich sind die Ergebnisse dieser Untersuchung schon allein über die Beschränkung auf kleine und mittlere Unternehmen begrenzt. Dabei ist weiter zu bedenken, dass die Entscheidung einer Bonuszahlung in einem Unternehmen eine vergleichsweise gehaltvolle Situation darstellt, die viele Anknüpfungspunkte zu möglichen realen Situationen aufweist. Das hat zur Folge, dass diese spezielle Handlung von einer Reihe institutioneller Merkmale im beruflichen Kontext der Probanden beeinflusst werden kann. So könnte man in weiteren Studien untersuchen, inwieweit z.B. die Lohnpolitik, die reale Gewinnsituation des Unternehmens und auch die Frage, ob bereits Boni gezahlt worden sind, die Entscheidungen der Probanden beeinflussen. 
Das Ziel der Untersuchung bestand jedoch auch nicht in der validen Bestimmung absoluter Bonuszahlungen in Unternehmen, sondern in der Identifizierung relevanter Prädiktoren sowie deren relativer Gewichte in verschiedenen situativen Entscheidungsumwelten. Der Vorzug unserer Vorgehensweise liegt dabei vor allem in der Rekrutierung einer interessanten Feldstichprobe, deren Entscheidungen im Kontext ihres speziellen institutionellen Feldes experimentell untersucht werden konnte. Aufgrund der Möglichkeit zur Variation und Randomisierung auf mehreren Ebenen (Within-Design auf der Vignettenebene und BetweenDesign auf der Ebene der Entscheidungssituation) erlaubte erst der faktorielle Survey überhaupt einen angemessenen experimentellen Zugang zu unserer Fragestellung. Zugunsten einer auf diese Weise hergestellten höheren externen Validität mussten die beschriebenen Abstriche bei der Störvariablenkontrolle in Kauf genommen werden.

Trotz der diskutierten Einschränkungen in der Aussagekraft, die mit einem faktoriellen Survey verbunden sind, sehen wir sehr große Vorteile in dessen Anwendung zur Untersuchung von Fairnessnormen. Insbesondere dann, wenn das faktorielle Within Subject-Design mit einem Between Subject-Design gekoppelt wird, um unterschiedliche Entscheidungsumwelten zu variieren, können differenzierte Ergebnisse erreicht werden. Neben der einfacheren Rekrutierung von speziellen Feldstichproben erlaubt diese Vorgehensweise umfassend kontrollierte zusätzliche Messungen und stellt daher insgesamt eine bereichernde methodische Ergänzung zu Labor- und Feldexperimenten einerseits und zur Umfrage andererseits dar.

\section{Literatur}

Abraham, Martin / Christina Meyer (2012): Bedingungen des Erfahrungsaustausches in wirtschaftlichen Transaktionen: Das Beispiel der Reputation von Weiterbildungsanbietern, in: Zeitschrift für Soziologie 41, S. 166-181.

Adams, John S. (1965): Inequity in social exchange, in: Leonard Berkowitz (Hrsg.), Advances in experimental social psychology, New York / NY, S. 267-299.

Akerlof, George A. / Janet L. Yellen (1990): The fair wage-effort hypothesis and unemployment, in: Quarterly Journal of Economics 105, S. 255-283.

Almas, Ingvild / Alexander W. Cappelen / Erik O. Sorensen / Bertil Tungodden (2010): Fairness and the development of inequality acceptance, in: Science 328, S. 1176-1178.

Alves, Wayne M. / Peter H. Rossi (1978): Who should get what? Fairness judgments of distribution of earnings, in: American Journal of Sociology 84, S. 541-564.

Andreoni, James (1990): Impure altruism and donations to public goods: A theory of warm-glow giving, in: The Economic Journal 100, S. 464-477.

Auspurg, Katrin / Annette Jäckle (2012): First equals most important? Order effects in vignette-based measurement, ISER Working Paper 2012-01, University of Essex: Institute for Social and Economic Research (ISER).

Auspurg, Katrin / Thomas Hinz / Stefan Liebig / Carsten Sauer (2010): Wie unplausibel darf es sein? Der Einfluss von Designmerkmalen auf das Antwortverhalten in Faktoriellen Surveys, in: Hans-Georg Soeffner (Hrsg.), Unsichere Zeiten. Herausforderungen gesellschaftlicher Transformation. Verhandlungen des 34. Kongresses der Deutschen Gesellschaft für Soziologie in Jena 2008, Wiesbaden (CD Rom).

Auspurg, Katrin / Thomas Hinz / Stefan Liebig / Carsten Sauer (2009): Auf das Design kommt es an. Experimentelle Befunde zu komplexen Settings in Faktoriellen Surveys, in: SoFid Methoden und Instrumente der Sozialwissenschaften 2009 / 2, S. 23-39.

Barrera, Davide / Vincent Buskens (2007): Imitation and learning: A vignette experiment, in: International Sociology 22, S. 367-396. 
Beck, Michael / Karl-Dieter Opp (2001): Der faktorielle Survey und die Messung von Normen, in: Kölner Zeitschrift für Soziologie und Sozialpsychologie 53, S. 283-306.

Ben-Ner, Avner / Amit Kramer / Ori Levy (2005): Economic and hypothetical dictator game experiments: Incentive effects at the individual level, in: The Journal of Socio-Economics 5, S. 1775-1784.

Berger, Roger / Marta Burek / Christiane Saller (2009): Online-Vignettenexperimente. Methode und Anwendung auf spieltheoretische Analysen, in: Nikolaus Jackob / Harald Schoen / Thomas Zerback (Hrsg.), Sozialforschung im Internet. Methodologie und Praxis der Online-Befragung, Wiesbaden, S. 305-319.

Buskens, Vincent / Jeroen Weesie (2000): An experiment on the effects of embeddedness in trust situations. Buying a used car, in: Rationality and Society 12, S. 227-253.

Camerer, Colin / Ernst Fehr (2009): Measuring social norms and preferences using experimental games, in: Henrich et al. (Hrsg.): Foundations of human sociality, Cambridge, S. 55-95.

Camerer, Colin (2003): Behavioral Game Theory, Princeton / NJ.

Cappelen Alexander W. / Astrid Hole / Erik O. Sorensen / Bertil Tungodden (2007): The pluralism of fairness ideals: An experimental approach, in: American Economic Review 97, S. 818-827.

Charness, Gary B. / Luca Rigotti / Aldo Rustichini (2007): Individual behavior and group membership, in: American Economic Review 97, S. 1340-1352.

Coleman, James (1991): Grundlagen der Sozialtheorie, München.

Cook, Karen S. / Karen A. Hegtvedt (1983): Distributive justice, equity, and equality, in: Annual Review of Sociology 9, S. 217-241.

Diekmann, Andreas (2008): Soziologie und Ökonomie: Der Beitrag experimenteller Wirtschaftsforschung zur Sozialtheorie, in: Kölner Zeitschrift für Soziologie und Sozialpsychologie 60, S. 528-550.

Dülmer, Hermann (2007): Experimental plans in factorial surveys: Random or quota design?, in: Sociological Methods and Research 35, S. 382-409.

Eifler, Stefanie (2010): Validity of a factorial survey approach to the analysis of criminal behavior, in: Methodology: European Journal of Research Methods for the Behavioral and Social Sciences 6, S. 139-146.

Eifler, Stefanie (2007): Evaluating the validity of self-reported deviant behavior using vignette analyses, in: Quality and Quantity 41, S. 303-318.

Eifler, Stefanie / Knut Petzold (2014): Der Einfluss der Ausführlichkeit von Vignetten auf die Erfassung prosozialer Einstellungen. Ergebnisse zweier Split-Ballot Experimente, in: Soziale Welt 65, S. $247-270$.

Engel, Christoph (2011): Dictator games: a meta study, in: Experimental Economics 14, S. 583-610.

Falk, Armin / James J. Heckman (2009): Lab experiments are a major source of knowledge in the social sciences, in: Science 326, S. 535-538.

Fehr, Ernst / Herbert Gintis (2007): Human motivation and social cooperation: Experimental and analytical foundations, in: Annual Review of Sociology 33, S. 43-64.

Fehr, Ernst / John A. List (2002): The hidden costs and returns of incentives - Trust and trustworthiness among CEO's, Working Paper No. 134, Institute of Empirical Research in Economics, University of Zürich.

Fehr, Ernst / Klaus Schmidt (1999): A theory of fairness, competition and cooperation, in: Quarterly Journal of Economics 114, S. 817-868.

Feng Chunliang / Yi Luo / Ruolei Gu / Lucas S. Broster / Xueyi Shen / Tengxiang Tian / Yue-Jia Luo / Frank Krueger (2013): The flexible fairness: Equality, earned entitlement, and self-interest, in: PLoS ONE 8, S. 1-18. 
Fischbacher, Urs / Nadja Kairies / Ulrike Stefani (2009): Performance, productivity and fairness. An experiment on the distribution of joint production, working paper, abrufbar unter: http:// area1.bwl.uni-mannheim.de/fileadmin/files/area1/files/research seminar/hws2011/Performance_Productivity_and_Fairness.pdf, letztes Abrufdatum: 7.9.2014.

Franzen, Axel / Sonja Pointner (2012): Anonymity in the dictator game revisited, in: Journal of Economic Behavior and Organization, 81 S. 74-81.

Frey, Bruno. S. / Stephan Meier (2004): Social comparisons and pro-social behavior - Testing conditional cooperation in a field experiment, in: American Economic Review 94, S. 1717-1722.

Friedman, Daniel / Alessandra Cassar (2009): Economics lab: An intensive course in experimental economics, London.

Gneezy, Uri / John A. List (2006): Putting behavioral economics to work: Testing for gift exchange in labor markets using field experiments, in: Econometrica 74, S. 1365-1384.

Gouldner, Alvin W. (1960): The norm of reciprocity: A preliminary statement, in: American Sociological Review 25, S. 161-178.

Groß, Jochen / Christina Börensen (2009): Wie valide sind Verhaltensmessungen mittels Vignetten? Ein methodischer Vergleich von Faktoriellem Survey und Verhaltensbeobachtung, in: Peter Kriwy / Christiane Gross (Hrsg.), Klein aber fein! Quantitative Sozialforschung mit kleinen Fallzahlen. Forschung und Entwicklung in der Analytischen Soziologie, Wiesbaden, S. 149-178

Gückelhorn, Cathrin / Olaf Struck / Matthias Dütsch / Gesine Stephan (2014): Bonuszahlungen an Manager. Eine Szenarienanalyse zu Gerechtigkeitsurteilen von Beschäftigten, Working Paper, abrufbar unter: http://www.uni-bamberg.de/fileadmin/arbeitswiss/Arbeitspapiere/Working_Paper_11.pdf, letztes Abrufdatum: 7.9.2014.

Güth, Werner / Rolf Schmittberger / Bernd Schwarze (1982): An experimental analysis of ultimatum bargaining, in: Journal of Economic Behavior and Organization 4, S. 367-388.

Hegtvedt, Karen A. (1992): When is a distribution rule just?, in: Rationality and Society 4, S. 308-331.

Hobbes, Thomas ([1651]1981): Leviathan, Harmondsworth.

Hoffman, Elizabeth / Kevin McCabe / Vernon L. Smith (1996): Social distance and other-regarding behaviour in Dictator Games, in: The American Economic Review 86, S. 653-660.

Hoffman, Elizabeth / Matthew Spitzer (1985): Entitlements, rights, and fairness: An experimental examination of subjects' concepts of distributive justice, in: Journal of Legal Studies 15, S. 254-297.

Homans, George C. (1961): Social behavior: Its elementary forms, New York / NY.

Hume, David ([1739-1740]2008): An enquiry concerning human understanding, Oxford.

Jasso, Guillermina (2006): Factorial survey methods for studying beliefs and judgments, in: Sociological Methods and Research 34, S. 334-423.

Jungbauer-Gans, Monika / Roger Berger / Peter Kriwy (2005): Machen Kleider Leute? Ergebnisse eines Feldexperiments zum Verkäuferverhalten, in: Zeitschrift für Soziologie, 34 S. 311-322.

Kahneman, Daniel / Jack Knetsch / Richard Thaler (1986): Fairness as a constraint on profit seeking: Entitlements in the market, in: American Economic Review 76, S. 728-741.

Konow, James (2003): Which is the fairest one of all? A positive analysis of justice theories, in: Journal of Economic Literature 41, S. 1188-1239.

Krumpal, Ivar (2013): Determinants of social desirability bias in sensitive surveys: A literature review, in: Quality and Quantity 47, S. 2025-2047.

Kuhfeld, Warren F. (2010): Marketing research methods in SAS. Experimental design, choice, conjoint, and graphical techniques, SAS 9.2 Edition MR-2010, Cary / NC.

Lazear, Edward P. / Sherwin Rosen (1981): Rank-order tournaments as optimum labor contracts, in: Journal of Political Economy 89, S. 841-864. 
Levitt, Steven D. / John A. List (2007): What do laboratory experiments measuring social preferences reveal about the real world?, in: The Journal of Economic Perspectives 21, S. 153-174.

Liebe, Ulf / Andreas Tutic (2010): Status groups and altruistic behavior in dictator games, in: Rationality and Society 22, S. 353-380.

Liebig, Stefan / Steffen Mau (2005): Wann ist ein Steuersystem gerecht? Einstellungen zu allgemeinen Prinzipien der Besteuerung und zur Gerechtigkeit der eigenen Steuerlast, in: Zeitschrift für Soziologie 34, S. 468-491.

Lucas, Jeffrey W. (2003): Theory-testing, generalization, and the problem of external validity, in: Sociological Theory 21, S. 236-253.

Mäs, Michael / Kurt Mühler / Karl-Dieter Opp (2005): Wann ist man deutsch? Empirische Ergebnisse eines Faktoriellen Surveys, in: Kölner Zeitschrift für Soziologie und Sozialpsychologie 57, S. 112-134.

Mill, John S. ([1863]2000): Der Utilitarismus, Stuttgart.

Nisic, Natascha / Katrin Auspurg (2009): Faktorieller Survey und klassische Bevölkerungsumfrage im Vergleich - Validität, Grenzen und Möglichkeiten beider Ansätze, in: Peter Kriwy / Christiane Gross (Hrsg.), Klein aber fein! Quantitative Sozialforschung mit kleinen Fallzahlen. Forschung und Entwicklung in der Analytischen Soziologie, Wiesbaden, S. 211-245

Nowak, Martin A. / Karl Sigmund (2005): Evolution of indirect reciprocity, in: Nature 437, S. 1291-1298.

Oosterbeek, Hessel / Randolph Sloof / Gijs van de Kuilen (2004): Cultural differences in ultimatum game experiments: Evidence from a meta-analysis, in: Experimental Economics 7, S. 171-188.

Oxoby, Robert J. / John Spraggon (2008): Mine and yours. Property rights in dictator games, in: Journal of Economic Behavior and Organization 65, S. 703-713.

Pointner, Sonja / Axel Franzen (2014): Fairness, in: Norman Braun und Nicole Saam (Hrsg.): Handbuch Modellbildung und Simulation in den Sozialwissenschaften, Wiesbaden (im Erscheinen).

Pointner, Sonja (2012): Das Fairnesskalkül. Robustheit, Determinanten und externe Validität der Fairnessnorm, Wiesbaden.

Rabe-Hesketh, Sophia / Anders Skrondal (2008): Multilevel and longitudinal modelling using Stata, Texas / TX.

Raub, Werner / Vincent Buskens (2006): Spieltheoretische Modellierungen und Anwendungen in der Soziologie, in: Andreas Diekmann (Hrsg.), Methoden der Sozialforschung, Wiesbaden, S. 560-598.

Rawls, John ([1957]2002): Eine Theorie der Gerechtigkeit, Frankfurt / Main.

Rooks, Gerrit / Werner Raub / Robert Selten / Frits Tazelaar (2000): How inter-firm co-operation depends on social embeddedness: A vignette study, in: Acta Sociologica 43, S. 123- 137.

Rossi, Peter H. / Andy B. Anderson (1982): The factorial survey approach: An introduction, in: Rossi, Peter H. / Steven L. Nock (Hrsg.), Measuring social judgments. The factorial approach. Beverly Hills / CA, S. 15-67.

Rossi, Peter H. (1979): Vignette analysis: Uncovering the normative structure of complex judgments, in: Merton, Robert K. / James S. Coleman / Peter H. Rossi (Hrsg.), Qualitative and Quantitative Social Research: Papers in Honor of Paul F. Lazarsfeld, New York / NY, S. 176-186.

Sauer, Carsten / Katrin Auspurg / Thomas Hinz / Stefan Liebig (2011): The application of factorial survey in general population samples: The effect of respondent age and education on response times and response consistency, in: Survey Research Methods 5, S. 89-102.

Smith, Adam ([1759]1986): The theory of moral sentiments, Düsseldorf.

Steiner, Peter M. / Christiane Atzmüller (2006): Experimentelle Vignettendesigns in faktoriellen Surveys, in: Kölner Zeitschrift für Soziologie und Sozialpsychologie 58,S. 117-146.

Treiman, Donald (1977): Occupational prestige in comparative perspective, New York / NY. 
Tutic, Andreas / Ulf Liebe (2009): A Theory of status-mediated inequity aversion, in: Journal of Mathematical Sociology 33, S. 157-195.

Vieth, Manuela (2004): Reziprozität im Gefangenendilemma. Eine spieltheoretische Untersuchung mit Hilfe eines faktoriellen Online-Surveys, Arbeitsberichte des Instituts für Soziologie der Universität Leipzig 40.

Wallander, Lisa (2009): 25 years of factorial surveys in sociology: A review, in: Social Science Research 38, S. 505-520.

Willer, Robb (2009): Groups reward individual sacrifice, in: American Sociological Review 74, S. 23-43.

Wolbring, Tobias / Christiane Bozoyan / Dominik Langner (2013): "Links gehen, rechts stehen!“ Ein Feldexperiment zur Durchsetzung informeller Normen auf Rolltreppen, in: Zeitschrift für Soziologie 42, S. 239-258.

Yamagishi, Toshio / Toshiro Mifune (2008): Does shared group membership promote altruism?, in: Rationality and Society 20, S. 5-30.

Martin Wienhold Leibniz Universität Hannover Institut für Soziologie

Schneiderberg 50

30167 Hannover m.wienhold@ish.uni-hannover.de

Dr. Knut Petzold Katholische Universität Eichstätt-Ingolstadt Fachgebiet Soziologie Ostenstraße 26 85072 Eichstätt knut.petzold@ku.de 


\section{Anhang}

Tabelle 4: Regressionseffekte der Arbeitnehmermerkmale auf die Höhe der Bonuszahlung, nicht unterschieden nach Informationsbedingungen

(1)

\begin{tabular}{|c|c|}
\hline & Bonuszablun \\
\hline Alter (45 Jabres) & $\begin{array}{c}0.1278 \\
(0.1519)\end{array}$ \\
\hline Alter (60 Jshro) & $\begin{array}{c}0.4199^{\circ *} \\
(0.1498)\end{array}$ \\
\hline Gerchlecht (woiblieh) & $\begin{array}{l}-0.1310 \\
(0.1188)\end{array}$ \\
\hline \multicolumn{2}{|l|}{ Zugeborigkeit (Aleferens: $t$ Jahr) } \\
\hline Zugehōriglkeit (5 Jahre) & $\begin{array}{c}0,8650^{* * *}+ \\
(0,1601)\end{array}$ \\
\hline Zugehōeigikeit (10 Jahre) & $\begin{array}{c}1.517 \theta^{* * *} \\
(0.2101)\end{array}$ \\
\hline \multicolumn{2}{|l|}{ Leistang (Refereno: unter) } \\
\hline Leistung (durchsehnittlich) & $\begin{array}{c}0.6894^{* * *} \\
(0.1372)\end{array}$ \\
\hline Leistung (aber-) & $\begin{array}{c}2.1887^{* * *} \\
(0.2606)\end{array}$ \\
\hline Gewerkschaftamitgliedschaft & $\begin{array}{l}0.1039 \\
(0.1233)\end{array}$ \\
\hline Macht (zugunaten Arbeitgeber) & $\begin{array}{l}-0.2110 \\
(0.1115)\end{array}$ \\
\hline \multicolumn{2}{|l|}{ Beru/sposition (Aleferene: Fecharbeiter) } \\
\hline Meinter & $\begin{array}{c}0.5238 \times 2 \\
(0.1596)\end{array}$ \\
\hline leitender Angertelleer & $\begin{array}{c}0.4544^{*+} \\
(0.1658)\end{array}$ \\
\hline \multicolumn{2}{|c|}{ Informationstrodingung (Feferenz: intraneparent) } \\
\hline teiltraneparest & $\begin{array}{l}-0.0078 \\
(0.4985)\end{array}$ \\
\hline traneparent & $\begin{array}{c}0.5682 \\
(0.5811)\end{array}$ \\
\hline Konatante & $\begin{array}{c}1,7761^{* * *} \\
(0.4465)\end{array}$ \\
\hline $\begin{array}{l}\text { N(Beobscbtungen) } \\
\text { aigmsman } \\
\text { nigmane } \\
\text { rbo }\end{array}$ & $\begin{array}{c}861 \\
1.8948 \\
1.5155 \\
0.5945\end{array}$ \\
\hline$R^{2}$ Within & 0.3788 \\
\hline$R^{2}$ Between & 0.0117 \\
\hline$R^{2}$ Gecamt & 0.1717 \\
\hline
\end{tabular}


Tabelle 5: Situationsabhängige Regressionseffekte der Arbeitnehmermerkmale auf die Höhe der Bonuszahlung, einschließlich Interaktionseffekten

\begin{tabular}{|c|c|c|c|c|c|}
\hline & $\begin{array}{c}\text { (1) } \\
\text { intrasiapareat } \\
\end{array}$ & $\begin{array}{c}\text { \{2\} } \\
\text { Internkation intrank, vw. Amdere }\end{array}$ & $\begin{array}{c}\text { (3) } \\
\text { teiltranaparent }\end{array}$ & $\begin{array}{c}\text { (4) } \\
\text { Internktion Andere va, tranuparmat }\end{array}$ & $\begin{array}{c}\text { (5) } \\
\text { trankparent }\end{array}$ \\
\hline \multicolumn{6}{|l|}{ Alter (R-genens: 30 Jober) } \\
\hline Alter (45 Jahre) & $\begin{array}{l}0.2879 \\
(0.3117)\end{array}$ & $\begin{array}{l}-0.2306 \\
(0.3513)\end{array}$ & $\begin{array}{l}-0.1590 \\
(0.2607)\end{array}$ & $\begin{array}{l}0.35-47 \\
(0.2865)\end{array}$ & $\begin{array}{l}0.3805 \\
(0.1973)\end{array}$ \\
\hline Altor (60 Jalure) & $\begin{aligned} 0.1044 \\
(0.1881)\end{aligned}$ & $\begin{array}{c}0.4560 \\
(0.2669)\end{array}$ & $\begin{array}{l}0.7162^{* *} \\
(0.2506)\end{array}$ & $\begin{array}{l}0.0847 \\
(0.3402)\end{array}$ & $\begin{array}{c}0.4911 \\
(0.2908)\end{array}$ \\
\hline Gesechlecht (weiblieb) & $\begin{array}{l}-0.1208 \\
(0.1772)\end{array}$ & $\begin{array}{l}-0.0239 \\
(0.2327)\end{array}$ & $\begin{array}{c}0.0538 \\
(0.3233)\end{array}$ & $\begin{array}{l}-0.2833 \\
(0.2142\rangle\end{array}$ & $\begin{array}{c}-0.3075 * * \\
(0.1157)\end{array}$ \\
\hline \multicolumn{6}{|l|}{ 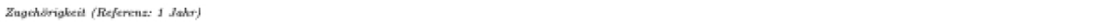 } \\
\hline Zugebiörigkenit (5 Jahre) & $\frac{0.8688 * \cdots}{(0.2139)}$ & $\begin{array}{l}-0.0914 \\
(0.2986)\end{array}$ & $\begin{array}{l}1.087200 \\
(0.3539)\end{array}$ & $\begin{array}{l}-0.3821 \\
(0.3131)\end{array}$ & $\frac{0.6240^{*}}{(0.2721)}$ \\
\hline Zugobürigkeit (10 Jahre) & $\begin{array}{c}1.7799^{*} \cdots \\
(0.3924)\end{array}$ & $\begin{array}{l}-0.4003 \\
(0.4588)\end{array}$ & $\begin{array}{c}1.4245^{* \ldots *} \\
(0.3885)\end{array}$ & $\begin{array}{l}-0.34856 \\
(0.40985)\end{array}$ & $\underset{\substack{1.2037^{* \cdots} \\
(0.2721)}}{ }$ \\
\hline \multicolumn{6}{|l|}{ Letistung (Reforease aster-) } \\
\hline Leistung (durebechnittlich) & $\begin{array}{l}0,8295 * * \\
(0.3124)\end{array}$ & $\begin{array}{l}-0,1780 \\
(0.3430)\end{array}$ & $\begin{array}{l}0.7651^{* * *} \\
(0.2101)\end{array}$ & $\begin{array}{l}-0.2652 \\
(0.2556)\end{array}$ & $\begin{array}{l}0.5390^{* *} \\
(0.1653)\end{array}$ \\
\hline Leistung (aber-) & $\begin{array}{l}2.4424 * \cdots \\
(0.4987)\end{array}$ & $\begin{array}{l}-0.3744 \\
(0.5832)\end{array}$ & $\begin{array}{l}2.0630 * * * \\
(0.4958)\end{array}$ & $\begin{array}{l}-1,1199^{\circ+*} \\
(0.4636)\end{array}$ & $\begin{array}{c}1.4909 * * * \\
(0.2939)\end{array}$ \\
\hline Gewerkschaftsmitgliedschat & $\begin{array}{c}0.0658 \\
(0.2330)\end{array}$ & $\begin{array}{l}0.0315 \\
(0.2759)\end{array}$ & $\begin{array}{c}0.3893 \\
(0.2420)\end{array}$ & $\begin{array}{l}-0.3080 \\
(0.2363)\end{array}$ & $\begin{array}{l}-0.0710 \\
(0.1693)\end{array}$ \\
\hline Macht (xugunsten Arbeitgeber) & $\begin{array}{l}-0.3787 \\
(0.2005)\end{array}$ & $\begin{array}{l}0.2300 \\
(0.2419)\end{array}$ & $\begin{array}{l}-0.4342 \\
(0.2256)\end{array}$ & $\begin{array}{l}0.4564^{*} \\
(0.2058)\end{array}$ & $\begin{array}{l}0.0661 \\
(0.1357)\end{array}$ \\
\hline \multicolumn{6}{|l|}{ 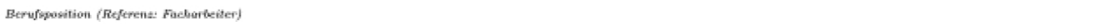 } \\
\hline Bertaf (Meinter) & $\begin{array}{c}0.3036 \\
(0.3958)\end{array}$ & $\begin{array}{l}0.3139 \\
(0.3766)\end{array}$ & $\begin{array}{l}0.6117^{*} \\
(0.2454)\end{array}$ & $\begin{array}{c}0.0172 \\
(0.2006)\end{array}$ & $\begin{array}{l}0.5170^{*} \\
(0.2056)\end{array}$ \\
\hline Beruf (leätender Angeatentenr) & $\begin{array}{l}0.1848 \\
(0.3348)\end{array}$ & $\begin{array}{l}0.3844 \\
(0.3849)\end{array}$ & $\begin{array}{l}0.8184^{* *} \\
(0.2930)\end{array}$ & $\begin{array}{l}-0.2800 \\
(0.3145)\end{array}$ & $\begin{array}{l}0.2841 \\
\langle 0.2206)\end{array}$ \\
\hline Kongtante & $\begin{array}{l}1.8606 * * \\
(0.5752)\end{array}$ & & $\begin{array}{l}1.2817^{*} \\
(0.4985)\end{array}$ & & $\begin{array}{c}2.7507^{-10 .} \\
(0.4951)\end{array}$ \\
\hline $\begin{array}{l}\text { N (Beabachtungen) } \\
\text { zigman,ne } \\
\text { xigran,en }\end{array}$ & $\begin{array}{l}\quad 861 \\
1.8796 \\
1.5126\end{array}$ & & $\begin{array}{l}861 \\
1.4760 \\
1.4971\end{array}$ & & $\begin{array}{l}861 \\
\begin{aligned} 8.8508 \\
1.4982\end{aligned}\end{array}$ \\
\hline 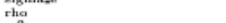 & $0.60 \% 0$ & & 0.4110 & & 0.0041 \\
\hline$R^{2}$ Within & 39 & & .40 & & .40 \\
\hline$R^{2}$ Between & .03 & & .01s & & .002 \\
\hline$R^{2}$ Genament & .17 & & .175 & & .193 \\
\hline
\end{tabular}

Tabelle 6: Anzahl fehlender Angaben zu Bonuszahlungen

\begin{tabular}{ccc}
\hline Missings & Häufigkeit & Anteil \\
\hline 0 & 64 & 37.4 \\
1 & 2 & $1.2 \%$ \\
2 & 3 & $1.8 \%$ \\
3 & 1 & $0.6 \%$ \\
5 & 1 & $0.6 \%$ \\
7 & 1 & $0.6 \%$ \\
8 & 2 & $1.2 \%$ \\
9 & 2 & $1.2 \%$ \\
10 & 1 & $0.6 \%$ \\
11 & 4 & $2.3 \%$ \\
12 & 90 & $52.6 \%$ \\
Total & 171 & $100.0 \%$ \\
\hline
\end{tabular}

